\title{
Effect of Treatments (Lemon, Mustard and Garlic) on the Minerals Value of Smoked Hilsa (Tenualosa ilisha) During Storage Period
}

\author{
Mohajira Begum ${ }^{1,3, \text { *, Shuva Bhowmik², Farha Matin Juliana }}{ }^{3}$, Md. Sabir Hossain ${ }^{3}$ \\ ${ }^{1}$ Fish Technology Research Section, Institute of Food Science and Technology (IFST), BCSIR, Dhaka, Bangladesh \\ ${ }^{2}$ Department of Fisheries Technology, Bangladesh Agricultural University, Mymensingh, Bangladesh \\ ${ }^{3}$ Department of Biochemistry \& Molecular Biology, Jahangirnagar University, Savar, Dhaka, Bangladesh
}

\section{Email address:}

Mohajira10@yahoo.com (M. Begum), shuva_bhowmik@yahoo.com (S. Bhowmik), farhamatin@juniv.edu (F. M. Juliana), sabirhossain@juniv.edu (M. S. Hossain)

${ }^{*}$ Corresponding author

\section{To cite this article:}

Mohajira Begum, Shuva Bhowmik, Farha Matin Juliana, Md. Sabir Hossain. Effect of Treatments (Lemon, Mustard and Garlic) on the Minerals Value of Smoked Hilsa (Tenualosa ilisha) During Storage Period. American Journal of Life Sciences.

Vol. 4, No. 5, 2016, pp. 133-138. doi: 10.11648/j.ajls.20160405.16

Received: August 31, 2016; Accepted: September 9, 2016; Published: October 27, 2016

\begin{abstract}
The study was designed to investigate the effect of both the duration of storage and treatments (lemon, mustrard and garlic) methods on minerals ( $\mathrm{Ca}, \mathrm{P}, \mathrm{Fe}, \mathrm{Mn}, \mathrm{Cu}, \mathrm{Mg}$ and $\mathrm{Zn}$ ) value of smoked hilsa in six selected regions (Chandpur, Barisal, Patuakhali, Bhola, Shariatpur and Cox's Bazar). Minerals (Ca, P, Fe, Mn, Cu, Mg and Zn) composition was increased by treatments but gradually decreased variedly with duration of storage smoked hilsa. The calcium $(\mathrm{Ca})$, phosphorus $(\mathrm{P})$, iron $(\mathrm{Fe})$, manganese $(\mathrm{Mn})$, copper $(\mathrm{Cu})$ magnesium $(\mathrm{Mg})$ and zinc $(\mathrm{Zn})$ were found $910.55 \pm 11.79$ to $853.57 \pm 11.09 \mathrm{mg} / 100 \mathrm{~g}$, $228.53 \pm 4.37$ to $83.60 \pm 8.75 \mathrm{mg} / 100 \mathrm{~g}, 12.45 \pm 0.97$ to $7.07 \pm 1.85 \mathrm{mg} / 100 \mathrm{~g}, 21.58 \pm 0.48$ to $7.89 \pm 0.75 \mathrm{mg} / 100 \mathrm{~g}, 3.15 \pm 0.16$ to $0.90 \pm 0.05 \mathrm{mg} / 100 \mathrm{~g}, 60.54 \pm 10.66$ to $36.31 \pm 1.85 \mathrm{mg} / 100 \mathrm{~g}$ and $2.97 \pm 0.10$ to $0.92 \pm 0.02 \mathrm{mg} / 100 \mathrm{~g}$ respectively. Generally, the minerals reduced more in the first month of storage. The results suggest that storage period to be a negative effect on the minearls values of smoked hilsa. Hence, smoked hilsa should be consumed while fresh and if it is to be stored, the period must be as short as possible to provide optimal levels of minerals.
\end{abstract}

Keywords: Lemon, Mustard, Garlic, Smoked Hilsa and Minerals

\section{Introduction}

Minerals are essential nutrients, they are components of many enzymes and metabolism, and contribute also to the growth of the fish [1]. Minerals such as iron (Fe), copper $(\mathrm{Cu})$ and manganese $(\mathrm{Mn})$ are essential and play important roles in biological systems [2]. Fish is very rich sources of various mineral components. The total content of minerals in raw flesh of marine fish and invertebrates is in the range of $0.6-1.5 \%$ of wet weight [3]. However, variation in mineral composition of fish can occur due to seasonal and biological differences (species, size, dark/white muscle, age, sex and sexual maturity), area of catch, processing method, food source and environmental conditions (water chemistry, salinity, temperature and contaminant) [4-6]. Fish energy depots in the forms of lipids that are indicate the quality of fish. The fish oil contain high amount of polyunsaturated fatty acid that reduce the serum cholesterol to prevent a number of coronary heart diseases. Fish meat is also a rich source of minerals and the most abundant micro-elements are iron $(\mathrm{Fe})$ and copper $(\mathrm{Cu})$ [7]. These minerals are generally higher in marine fish than in fresh water fish [8].

The hilsa (Tenualosa ilisha) of the subfamily Alosinae, family Clupeidae, order Clupeiformes, is one of the most important tropical fishes of the Indo-Pacific region and has occupied a top position among the edible fishes owing to its taste, flavor and culinary properties. Hilsa is locally called Ilish and the fish has been designated as the national fish of Bangladesh. Hilsa is one of the commercially important 
anadromous fish high in fat and hailed as 'macher raja-ilish' in Bangladesh. Among the available fish resources, it is preferred and regarded as a blessing for their incredible flavor, taste and delicacy. Although Bangladeshi people consume hilsa because of its abundance, they have a little knowledge on the nutritional values of hilsa. This study was therefore undertaken to create a base line data on the mineral composition of the smoked hilsa during different storage condition.

\section{Materials and Methods}

\subsection{Collection of Fish}

The experimental hilsa fish was collected from six selected regions (Chandpur, Barisal, Patuakhali, Bhola, Shariatpur and Cox's Bazar) of Bangladesh. The fish samples were transported to the laboratory in sterile polythene bags to avoid any type of microbial contamination.

\subsection{Smoking Procedure}

The hilsa samples were killed, gutted and washed thoroughly with clean water and were laid on the racks of the smoking kiln (model: ELC 1600). Heat was generated by the burning of charcoal from logs of wood and smoking of the samples was carried out at $60-70^{\circ} \mathrm{C}$ for 12 hours. After smoking, the products were packed in polythene bags to reduce pest/microbial infestation and kept in the refrigerator (Hajer Thermocool) at $1-5^{\circ} \mathrm{C}$ for 60 days.

\subsection{Mineral Elements Analysis}

The preparation of samples for mineral elements analysis followed a method described by AOAC [9]. Approximately 5 $\mathrm{g}$ of sample was weighed into acid-washed crucible and dried in oven $105^{\circ} \mathrm{C}$ for one day. Dried samples were then digested in furnace oven at $550^{\circ} \mathrm{C}$ overnight. The ash was digested in $5 \mathrm{ml}$ of $65 \%$ nitric acid $\left(\mathrm{HNO}_{3}\right)$ by boiling for about two minutes and cooling to room temperature. The cooled solution was filtered through Whatman filter paper (No. 41) and made up to $25 \mathrm{ml}$ with $65 \%$ nitric acid [9]. A $10 \mathrm{ml}$ were transferred into $15 \mathrm{ml}$ polypropylene test tube for injection into inductively-coupled plasma-optical emission spectrometer (ICP-OES) (Perkin Elmer, USA). Samples were then analyzed for its micro minerals content calcium $(\mathrm{Ca})$, phosphorus $(\mathrm{P})$, iron $(\mathrm{Fe})$, manganese $(\mathrm{Mn})$, copper $(\mathrm{Cu})$, magnesium $(\mathrm{Mg})$ and Zinc $(\mathrm{Zn})$. Sample blank (65\% nitric acid) was analysed together with each batch of samples.

\subsection{Data Analyses}

After experiment, data were sorted, edited and encoded. All the collected data were summarized, scrutinized; tabulated and carefully subjected to the descriptive analyses using the computer software MS Word, Microsoft Office Excel 2007 and XL-stat version 16 for DMRT to understand the differences of the variables.

\section{Results and Discussion}

The minerals content of the smoked hilsa was gradually decreased during the storage period (Table 1-7). The ranged of calcium content lemon, mustard and garlic treatments smoked hilsa were found $930.11 \pm 9.42$ to $867.22 \pm 11.04$, $954.29 \pm 5.32$ to $886.56 \pm 11.04$ and $990.18 \pm 8.31$ to $895.29 \pm 19.53 \mathrm{mg} / 100 \mathrm{~g}$ respectively in storage condition. Similarly the phosphorus (247.19 \pm 8.342 to $89.70 \pm 6.54$, $277.15 \pm 5.42$ to $120.96 \pm 3.28$ and $290.87 \pm 5.48$ to $155.30 \pm 4.38$ $\mathrm{mg} / 100 \mathrm{~g})$, iron $\quad(12.50 \pm 0.97 \quad$ to $7.19 \pm 0.09, \quad 12.65 \pm 0.98$ to $7.46 \pm 0.48$ and $12.80 \pm 0.09$ to $7.77 \pm 0.48 \mathrm{mg} / 100 \mathrm{~g})$, manganese $(17.71 \pm 0.83$ to $8.54 \pm 0.16, \quad 19.56 \pm 0.85$ to $8.56 \pm 0.79$ and $14.40 \pm 0.67$ to $8.87 \pm 0.64 \mathrm{mg} / 100 \mathrm{~g}$ ), copper $(2.22 \pm 0.17$ to $1.09 \pm 0.08,2.20 \pm 0.05$ to $1.21 \pm 0.03$ and $2.66 \pm 0.32$ to $0.92 \pm 0.18 \mathrm{mg} / 100 \mathrm{~g})$, magnesium $(62.92 \pm 5.50$ to $41.95 \pm 6.87,63.90 \pm 1.84$ to $44.65 \pm 4.53$ and $64.82 \pm 5.45$ to $46.87 \pm 1.97 \mathrm{mg} / 100 \mathrm{~g})$ and zinc $(3.92 \pm 0.14$ to $1.11 \pm 0.09$, $2.77 \pm 0.19$ to $1.23 \pm 0.23$ and $2.90 \pm 0.01$ to $0.93 \pm 0.19$ $\mathrm{mg} / 100 \mathrm{~g}$ ) value of smoked hilsa were slowly decreased in storage condition. The variations recorded in the concentration of minerals in the hilsa fish is generally expected because of potential variations in the rate with which they are available in the water body and the ability to absorb these inorganic elements [10]. It was also observed that reduction was greater in the first month of storage. This can be attributed to high microbial activities on the fish during the first month of storage due to the organisms that are naturally found on the fresh fish [10]. After the fish is stored for some time, the microbial activity reduces as their activity is lowered by temperature and some of these microbes die with increasing duration of storage [11]. Variations recorded in the values of calcium could be either due to their diet selection or an increase in the proportion of bone to flesh as the fishes grows [12]. Although some previous studies [7] reported higher levels of calcium in some fish species, the results of the present study seem to disagree with their findings. This can be attributed to the differences in treatments (lemon, mustard and garlic), size, weight and storage period of the smoked hilsa.

Table 1. Calcium content ( $\mathrm{mg} / 100 \mathrm{~g})$ in smoked hilsa during storage period.

\begin{tabular}{|c|c|c|c|c|c|c|c|}
\hline Treatments & Days & Chandpur & Barisal & Patuakhali & Bhola & Shariatpur & Cox's Bazar \\
\hline \multirow{5}{*}{ Control } & 0 (fresh) & $910.55 \pm 11.79^{\mathrm{e}}$ & $881.39 \pm 6.94^{\mathrm{e}}$ & $909.50 \pm 11.32^{\mathrm{e}}$ & $884.26 \pm 8.28^{\mathrm{e}}$ & $908.23 \pm 13.52^{\mathrm{e}}$ & $870.72 \pm 6.84^{\mathrm{e}}$ \\
\hline & 15 & $904.43 \pm 10.76^{\mathrm{d}}$ & $876.64 \pm 7.39^{d}$ & $894.58 \pm 17.83^{\mathrm{d}}$ & $877.93 \pm 9.36^{\mathrm{d}}$ & $897.53 \pm 17.53^{d}$ & $89.32 \pm 13.42^{\mathrm{d}}$ \\
\hline & 30 & $899.05 \pm 9.74^{\mathrm{c}}$ & $871.13 \pm 9.88^{c}$ & $877.94 \pm 5.38^{c}$ & $871.64 \pm 10.38^{c}$ & $888.94 \pm 12.95^{\mathrm{c}}$ & $865.82 \pm 7.13^{c}$ \\
\hline & 45 & $892.14 \pm 18.29^{b}$ & $867.43 \pm 10.43^{b}$ & $865.23 \pm 9.27^{\mathrm{b}}$ & $861.56 \pm 7.32^{b}$ & $871.63 \pm 8.54^{b}$ & $856.52 \pm 9.41^{b}$ \\
\hline & 60 & $885.22 \pm 8.53^{\mathrm{a}}$ & $863.95 \pm 3.79^{\mathrm{a}}$ & $857.69 \pm 9.32^{\mathrm{a}}$ & $853.57 \pm 11.09^{\mathrm{a}}$ & $859.90 \pm 9.17^{\mathrm{a}}$ & $848.29 \pm 8.59^{\mathrm{a}}$ \\
\hline Lemon & 0 (fresh) & $930.11 \pm 9.42^{\mathrm{e}}$ & $900.57 \pm 17.52^{\mathrm{e}}$ & $924.75 \pm 5.37^{\mathrm{e}}$ & $905.33 \pm 17.53^{\mathrm{e}}$ & $921.50 \pm 8.32^{\mathrm{e}}$ & $898.53 \pm 15.32^{e}$ \\
\hline
\end{tabular}




\begin{tabular}{|c|c|c|c|c|c|c|c|}
\hline Treatments & Days & Chandpur & Barisal & Patuakhali & Bhola & Shariatpur & Cox's Bazar \\
\hline \multirow{8}{*}{ Mustard } & 15 & $924.42 \pm 8.54^{\mathrm{d}}$ & $893.23 \pm 15.09^{d}$ & $918.94 \pm 7.42^{\mathrm{d}}$ & $899.67 \pm 19.49^{d}$ & $917.43 \pm 11.72^{\mathrm{d}}$ & $888.88 \pm 13.92^{d}$ \\
\hline & 30 & $919.65 \pm 17.43^{\mathrm{c}}$ & $886.54 \pm 8.42^{\mathrm{c}}$ & $912.32 \pm 17.48^{\mathrm{c}}$ & $893.45 \pm 21.03^{\mathrm{c}}$ & $909.21 \pm 12.69^{\mathrm{c}}$ & $879.53 \pm 17.09^{c}$ \\
\hline & 45 & $914.67 \pm 18.53^{b}$ & $877.98 \pm 15.48^{b}$ & $899.90 \pm 15.48^{b}$ & $890.89 \pm 7.38^{\mathrm{b}}$ & $893.54 \pm 7.93^{\mathrm{b}}$ & $873.56 \pm 4.70^{b}$ \\
\hline & 60 & $910.46 \pm 15.42^{\mathrm{a}}$ & $873.80 \pm 11.98^{\mathrm{a}}$ & $892.77 \pm 19.63^{\mathrm{a}}$ & $888.25 \pm 9.32^{\mathrm{a}}$ & $888.47 \pm 7.38^{\mathrm{a}}$ & $867.22 \pm 11.04^{\mathrm{a}}$ \\
\hline & 0 (fresh) & $944.93 \pm 10.64^{\mathrm{e}}$ & $918.13 \pm 3.98^{\mathrm{e}}$ & $954.29 \pm 5.32^{\mathrm{e}}$ & $920.99 \pm 8.39^{\mathrm{e}}$ & $935.98 \pm 9.36^{\mathrm{e}}$ & $907.24 \pm 15.82^{\mathrm{e}}$ \\
\hline & 15 & $939.76 \pm 13.58^{d}$ & $910.57 \pm 11.79^{d}$ & $946.53 \pm 15.69^{d}$ & $914.09 \pm 5.48^{\mathrm{d}}$ & $929.45 \pm 11.94^{\mathrm{d}}$ & $904.43 \pm 17.32^{\mathrm{d}}$ \\
\hline & 30 & $933.87 \pm 12.86^{\mathrm{c}}$ & $897.45 \pm 8.53^{c}$ & $935.87 \pm 3.79^{c}$ & $908.34 \pm 6.52^{\mathrm{c}}$ & $923.16 \pm 16.49^{c}$ & $897.43 \pm 16.41^{\mathrm{c}}$ \\
\hline & 45 & $927.84 \pm 5.89^{\mathrm{b}}$ & $891.76 \pm 8.27^{b}$ & $926.76 \pm 16.83^{b}$ & $903.45 \pm 7.39^{\mathrm{b}}$ & $911.56 \pm 13.21^{\mathrm{b}}$ & $890.45 \pm 10.27^{b}$ \\
\hline & 60 & $920.57 \pm 9.42^{\mathrm{a}}$ & $887.70 \pm 7.48^{\mathrm{a}}$ & $918.52 \pm 7.42^{\mathrm{a}}$ & $899.52 \pm 10.03^{\mathrm{a}}$ & $900.24 \pm 7.92^{\mathrm{a}}$ & $886.56 \pm 11.04^{\mathrm{a}}$ \\
\hline \multirow{5}{*}{ Garlic } & 0 (fresh) & $965.53 \pm 17.43^{\mathrm{e}}$ & $990.18 \pm 8.31^{\mathrm{e}}$ & $957.12 \pm 4.38^{\mathrm{e}}$ & $940.18 \pm 5.93^{\mathrm{e}}$ & $962.67 \pm 5.11^{\mathrm{e}}$ & $918.51 \pm 19.03^{\mathrm{e}}$ \\
\hline & 15 & $957.62 \pm 19.73^{d}$ & $983.65 \pm 12.11^{\mathrm{d}}$ & $953.45 \pm 7.48^{\mathrm{d}}$ & $932.91 \pm 7.49^{d}$ & $953.56 \pm 5.99^{d}$ & $911.45 \pm 23.92^{\mathrm{d}}$ \\
\hline & 30 & $951.80 \pm 11.94^{\mathrm{c}}$ & $970.07 \pm 5.31^{\mathrm{c}}$ & $948.98 \pm 10.04^{c}$ & $921.63 \pm 23.34^{c}$ & $947.21 \pm 7.52^{\mathrm{c}}$ & $904.78 \pm 18.54^{\mathrm{c}}$ \\
\hline & 45 & $943.87 \pm 6.04^{b}$ & $957.98 \pm 3.84^{\mathrm{b}}$ & $935.43 \pm 6.38^{b}$ & $911.67 \pm 11.73^{b}$ & $938.92 \pm 5.18^{b}$ & $901.23 \pm 10.49^{b}$ \\
\hline & 60 & $933.40 \pm 13.29^{\mathrm{a}}$ & $945.73 \pm 16.42^{\mathrm{a}}$ & $925.81 \pm 8.52^{\mathrm{a}}$ & $904.70 \pm 7.32^{\mathrm{a}}$ & $930.25 \pm 10.21^{\mathrm{a}}$ & $895.29 \pm 19.53^{\mathrm{a}}$ \\
\hline
\end{tabular}

*The values in the same column having similar superscripts do not differ significantly $(\mathrm{p}<0.05)$

Table 2. Phosphorus content ( $\mathrm{mg} / 100 \mathrm{~g})$ in smoked hilsa during storage period.

\begin{tabular}{|c|c|c|c|c|c|c|c|}
\hline Treatments & Days & Chandpur & Barisal & Patuakhali & Bhola & Shariatpur & Cox's Bazar \\
\hline \multirow{5}{*}{ Control } & 0 (fresh) & $130.56 \pm 5.87^{\mathrm{e}}$ & $228.53 \pm 4.37^{\mathrm{e}}$ & $164.67 \pm 5.98^{\mathrm{e}}$ & $209.02 \pm 4.87^{\mathrm{e}}$ & $114.95 \pm 6.37^{\mathrm{e}}$ & $118.25 \pm 5.87^{\mathrm{e}}$ \\
\hline & 15 & $126.56 \pm 5.73^{d}$ & $223.56 \pm 5.94^{d}$ & $154.84 \pm 4.38^{\mathrm{d}}$ & $204.56 \pm 5.38^{d}$ & $103.46 \pm 5.05^{\mathrm{d}}$ & $113.37 \pm 5.09^{d}$ \\
\hline & 30 & $115.76 \pm 8.63^{\mathrm{c}}$ & $216.49 \pm 6.43^{\mathrm{c}}$ & $143.98 \pm 6.32^{\mathrm{c}}$ & $197.65 \pm 2.84^{\mathrm{c}}$ & $94.43 \pm 4.86^{\mathrm{c}}$ & $105.87 \pm 7.98^{\mathrm{c}}$ \\
\hline & 45 & $105.67 \pm 9.37^{b}$ & $208.45 \pm 7.32^{\mathrm{b}}$ & $134.76 \pm 5.46^{\mathrm{b}}$ & $189.76 \pm 7.93^{b}$ & $89.09 \pm 9.63^{\mathrm{b}}$ & $97.88 \pm 5.47^{\mathrm{b}}$ \\
\hline & 60 & $100.95 \pm 7.85^{\mathrm{a}}$ & $202.05 \pm 8.52^{\mathrm{a}}$ & $124.11 \pm 5.19^{\mathrm{a}}$ & $182.13 \pm 6.39^{\mathrm{a}}$ & $83.60 \pm 8.75^{\mathrm{a}}$ & $94.29 \pm 6.78^{\mathrm{a}}$ \\
\hline \multirow{5}{*}{ Lemon } & 0 (fresh) & $164.67 \pm 9.34^{\mathrm{e}}$ & $247.19 \pm 8.342^{\mathrm{e}}$ & $180.91 \pm 3.86^{\mathrm{e}}$ & $221.25 \pm 8.42^{\mathrm{e}}$ & $116.90 \pm 4.67^{\mathrm{e}}$ & $133.90 \pm 4.63^{\mathrm{e}}$ \\
\hline & 15 & $156.49 \pm 11.73^{\mathrm{d}}$ & $237.81 \pm 8.50^{\mathrm{d}}$ & $173.82 \pm 2.85^{\mathrm{d}}$ & $216.38 \pm 7.49^{\mathrm{d}}$ & $111.45 \pm 10.42^{\mathrm{d}}$ & $129.63 \pm 4.97^{\mathrm{d}}$ \\
\hline & 30 & $147.82 \pm 6.39^{c}$ & $226.45 \pm 10.32^{\mathrm{C}}$ & $167.54 \pm 3.19^{c}$ & $209.76 \pm 7.33^{\mathrm{c}}$ & $104.35 \pm 4.38^{\mathrm{c}}$ & $124.34 \pm 8.95^{\mathrm{c}}$ \\
\hline & 45 & $137.84 \pm 3.49^{b}$ & $219.63 \pm 6.47^{\mathrm{b}}$ & $156.45 \pm 9.53^{\mathrm{b}}$ & $199.70 \pm 4.58^{\mathrm{b}}$ & $98.46 \pm 8.59^{b}$ & $117.45 \pm 2.09^{b}$ \\
\hline & 60 & $129.80 \pm 7.62^{\mathrm{a}}$ & $213.20 \pm 8.43^{\mathrm{a}}$ & $143.83 \pm 6.18^{\mathrm{a}}$ & $194.07 \pm 3.84^{\mathrm{a}}$ & $89.70 \pm 6.54^{\mathrm{a}}$ & $107.55 \pm 7.68^{\mathrm{a}}$ \\
\hline \multirow{5}{*}{ Mustard } & 0 (fresh) & $179.08 \pm 7.52^{\mathrm{e}}$ & $277.15 \pm 5.42^{\mathrm{e}}$ & $175.65 \pm 5.29^{\mathrm{e}}$ & $240.98 \pm 7.13^{\mathrm{e}}$ & $157.36 \pm 7.40^{\mathrm{e}}$ & $162.55 \pm 6.54^{\mathrm{e}}$ \\
\hline & 15 & $171.23 \pm 8.22^{\mathrm{d}}$ & $267.64 \pm 3.94^{\mathrm{d}}$ & $167.34 \pm 4.94^{\mathrm{d}}$ & $233.87 \pm 5.80^{\mathrm{d}}$ & $154.37 \pm 9.53^{\mathrm{d}}$ & $155.39 \pm 6.43^{\mathrm{d}}$ \\
\hline & 30 & $156.45 \pm 4.39^{c}$ & $256.45 \pm 11.05^{\mathrm{c}}$ & $149.65 \pm 9.49^{c}$ & $229.56 \pm 10.93^{\mathrm{c}}$ & $149.57 \pm 10.47^{\mathrm{c}}$ & $145.76 \pm 4.43^{\mathrm{c}}$ \\
\hline & 45 & $145.98 \pm 2.06^{\mathrm{b}}$ & $246.73 \pm 6.38^{b}$ & $135.67 \pm 7.16^{\mathrm{b}}$ & $223.76 \pm 6.28^{b}$ & $143.67 \pm 5.32^{\mathrm{b}}$ & $139.34 \pm 10.09^{b}$ \\
\hline & 60 & $138.91 \pm 7.93^{\mathrm{a}}$ & $231.92 \pm 2.74^{\mathrm{a}}$ & $120.96 \pm 3.28^{\mathrm{a}}$ & $215.14 \pm 4.82^{\mathrm{a}}$ & $138.07 \pm 7.68^{\mathrm{a}}$ & $133.97 \pm 10.44^{\mathrm{a}}$ \\
\hline \multirow{5}{*}{ Garlic } & 0 (fresh) & $200.55 \pm 2.99^{\mathrm{e}}$ & $290.87 \pm 5.48^{\mathrm{e}}$ & $190.43 \pm 10.74^{\mathrm{e}}$ & $262.59 \pm 4.94^{\mathrm{e}}$ & $179.89 \pm 4.98^{\mathrm{e}}$ & $193.11 \pm 4.35^{\mathrm{e}}$ \\
\hline & 15 & $189.54 \pm 4.83^{\mathrm{d}}$ & $284.78 \pm 2.96^{\mathrm{d}}$ & $182.65 \pm 3.85^{\mathrm{d}}$ & $257.45 \pm 3.84^{\mathrm{d}}$ & $170.98 \pm 5.97^{\mathrm{d}}$ & $189.62 \pm 6.87^{\mathrm{d}}$ \\
\hline & 30 & $177.46 \pm 3.95^{\mathrm{c}}$ & $279.45 \pm 5.93^{\mathrm{c}}$ & $173.67 \pm 5.98^{c}$ & $249.76 \pm 8.52^{\mathrm{c}}$ & $166.56 \pm 7.47^{\mathrm{c}}$ & $184.36 \pm 8.97^{\circ}$ \\
\hline & 45 & $169.87 \pm 6.83^{\mathrm{b}}$ & $271.56 \pm 4.68^{\mathrm{b}}$ & $169.62 \pm 8.75^{\mathrm{b}}$ & $244.66 \pm 1.05^{\mathrm{b}}$ & $162.48 \pm 6.53^{\mathrm{b}}$ & $179.47 \pm 4.79^{\mathrm{b}}$ \\
\hline & 60 & $163.15 \pm 2.94^{\mathrm{a}}$ & $266.51 \pm 3.83^{\mathrm{a}}$ & $162.90 \pm 10.26^{\mathrm{a}}$ & $240.15 \pm 3.64^{\mathrm{a}}$ & $155.30 \pm 4.38^{\mathrm{a}}$ & $175.11 \pm 9.80^{\mathrm{a}}$ \\
\hline
\end{tabular}

*The values in the same column having similar superscripts do not differ significantly $(p<0.05)$

We can also attribute the variations to the differences in the feeding behaviour. This observation was supported by the findings of [13] which showed that such variations in concentrations of these mineral elements of smoked hilsa was due to the chemical forms of the elements and their concentrations in the local environment. The result again do agree with the findings of [14] who observed that there was a slight change with respect to frozen period in fish minerals evaluated. This could be attributed to drip loss and dehydration that is associated with frozen storage [15]. In contrast to previous studies which only found a slight change in minerals, the changes in minerals composition were stronger in the present study. The small differences can be attributed to the short period of storage which was one month in the previous studies while the present study had the fish stored for two months. Generally, our findings seem to suggest that freezing also has a negative effect on the nutritional values of smoked hilsa. The reduction can be likened to the negative effects of freezing and storage period which including freezer burn, product dehydration, rancidity, drip loss and product bleaching which can have an overall negative effect on the quality of the frozen foods [16].

Table 3. Iron content ( $\mathrm{mg} / \mathrm{l} 00 \mathrm{~g})$ in smoked hilsa during storage period.

\begin{tabular}{|c|c|c|c|c|c|c|c|}
\hline Treatments & Days & Chandpur & Barisal & Patuakhali & Bhola & Shariatpur & Cox's Bazar \\
\hline \multirow{5}{*}{ Control } & 0 (fresh) & $7.36 \pm 1.87^{\mathrm{e}}$ & $12.45 \pm 0.97^{\mathrm{e}}$ & $10.75 \pm 1.13^{\mathrm{e}}$ & $8.91 \pm 2.09^{\mathrm{e}}$ & $7.77 \pm 0.58^{\mathrm{e}}$ & $7.53 \pm 1.64^{\mathrm{e}}$ \\
\hline & 15 & $7.28 \pm 1.25^{\mathrm{d}}$ & $11.43 \pm 0.58^{\mathrm{d}}$ & $10.49 \pm 1.07^{\mathrm{d}}$ & $7.785 \pm 0.96^{\mathrm{d}}$ & $7.71 \pm 0.74^{\mathrm{d}}$ & $7.39 \pm 0.68^{d}$ \\
\hline & 30 & $7.16 \pm 0.86^{c}$ & $11.42 \pm 0.74^{\mathrm{c}}$ & $10.24 \pm 0.64^{c}$ & $7.46 \pm 0.64^{\mathrm{c}}$ & $7.66 \pm 0.59^{c}$ & $7.11 \pm 0.84^{c}$ \\
\hline & 45 & $7.11 \pm 0.47^{b}$ & $11.41 \pm 0.86^{\mathrm{b}}$ & $9.99 \pm 0.37^{\mathrm{b}}$ & $7.34 \pm 0.64^{b}$ & $7.58 \pm 0.83^{b}$ & $6.98 \pm 0.78^{b}$ \\
\hline & 60 & $7.07 \pm 1.85^{\mathrm{a}}$ & $11.40 \pm 0.53^{\mathrm{a}}$ & $9.87 \pm 0.62^{\mathrm{a}}$ & $7.12 \pm 0.54^{\mathrm{a}}$ & $7.50 \pm 0.76^{\mathrm{a}}$ & $6.84 \pm 0.63^{\mathrm{a}}$ \\
\hline \multirow{3}{*}{ Lemon } & 0 (fresh) & $7.97 \pm 0.54^{\mathrm{e}}$ & $12.50 \pm 0.97^{\mathrm{e}}$ & $10.57 \pm 1.68^{\mathrm{e}}$ & $10.21 \pm 0.73^{\mathrm{e}}$ & $8.06 \pm 0.95^{\mathrm{e}}$ & $7.79 \pm 0.13^{\mathrm{e}}$ \\
\hline & 15 & $7.88 \pm 0.73^{d}$ & $11.49 \pm 0.36^{\mathrm{d}}$ & $10.45 \pm 0.96^{\mathrm{d}}$ & $10.16 \pm 0.63^{d}$ & $7.89 \pm 0.93^{\mathrm{d}}$ & $7.65 \pm 0.65^{d}$ \\
\hline & 45 & $7.45 \pm 0.75^{b}$ & $11.46 \pm 0.73^{\mathrm{b}}$ & $10.10 \pm 0.85^{\mathrm{b}}$ & $9.99 \pm 0.53^{\mathrm{b}}$ & $7.36 \pm 0.73^{b}$ & $7.11 \pm 0.89^{b}$ \\
\hline
\end{tabular}




\begin{tabular}{llllllll}
\hline Treatments & Days & Chandpur & Barisal & Patuakhali & Bhola & Shariatpur & Cox's Bazar \\
\hline \multirow{6}{*}{ Mustard } & 60 & $7.20 \pm 0.72^{\mathrm{a}}$ & $11.45 \pm 0.95^{\mathrm{a}}$ & $9.96 \pm 0.97^{\mathrm{a}}$ & $9.91 \pm 0.08^{\mathrm{a}}$ & $7.19 \pm 0.09^{\mathrm{a}}$ & $6.94 \pm 0.93^{\mathrm{a}}$ \\
& 0 (fresh) & $8.20 \pm 0.37^{\mathrm{c}}$ & $12.65 \pm 0.98^{\mathrm{e}}$ & $10.79 \pm 0.74^{\mathrm{e}}$ & $10.89 \pm 0.67^{\mathrm{e}}$ & $8.68 \pm 0.73^{\mathrm{e}}$ & $9.29 \pm 0.79^{\mathrm{e}}$ \\
& 15 & $7.96 \pm 0.17^{\mathrm{d}}$ & $12.49 \pm 1.09^{\mathrm{d}}$ & $10.67 \pm 0.97^{\mathrm{d}}$ & $10.59 \pm 0.84^{\mathrm{d}}$ & $8.37 \pm 0.87^{\mathrm{d}}$ & $9.01 \pm 0.62^{\mathrm{d}}$ \\
& 30 & $7.77 \pm 0.87^{\mathrm{c}}$ & $12.23 \pm 0.93^{\mathrm{c}}$ & $10.45 \pm 1.06^{\mathrm{c}}$ & $10.23 \pm 0.47^{\mathrm{c}}$ & $7.95 \pm 0.42^{\mathrm{c}}$ & $8.77 \pm 0.59^{\mathrm{c}}$ \\
& 45 & $7.67 \pm 1.07^{\mathrm{b}}$ & $11.98 \pm 1.03^{\mathrm{b}}$ & $10.19 \pm 1.09^{\mathrm{b}}$ & $9.87 \pm 1.52^{\mathrm{b}}$ & $7.88 \pm 0.52^{\mathrm{b}}$ & $8.46 \pm 1.63^{\mathrm{b}}$ \\
& 60 & $7.46 \pm 0.48^{\mathrm{a}}$ & $11.80 \pm 0.78^{\mathrm{a}}$ & $10.09 \pm 0.86^{\mathrm{a}}$ & $9.64 \pm 0.87^{\mathrm{a}}$ & $7.60 \pm 0.52^{\mathrm{a}}$ & $8.28 \pm 0.84^{\mathrm{a}}$ \\
& 0 (fresh) & $8.42 \pm 0.73^{\mathrm{e}}$ & $12.80 \pm 0.09^{\mathrm{a}}$ & $10.85 \pm 0.54^{\mathrm{e}}$ & $11.67 \pm 1.36^{\mathrm{e}}$ & $9.06 \pm 0.73^{\mathrm{e}}$ & $9.45 \pm 0.63^{\mathrm{e}}$ \\
Garlic & 15 & $8.34 \pm 0.28^{\mathrm{d}}$ & $12.67 \pm 0.13^{\mathrm{d}}$ & $10.77 \pm 0.38^{\mathrm{d}}$ & $11.02 \pm 0.63^{\mathrm{d}}$ & $8.88 \pm 0.48^{\mathrm{d}}$ & $9.14 \pm 0.73^{\mathrm{d}}$ \\
& 30 & $8.18 \pm 0.54^{\mathrm{c}}$ & $12.35 \pm 0.34^{\mathrm{c}}$ & $10.57 \pm 0.94^{\mathrm{c}}$ & $10.47 \pm 0.36^{\mathrm{c}}$ & $8.67 \pm 0.63^{\mathrm{c}}$ & $8.93 \pm 0.98^{\mathrm{c}}$ \\
& 45 & $7.94 \pm 0.96^{\mathrm{b}}$ & $12.11 \pm 0.54^{\mathrm{b}}$ & $10.34 \pm 0.38^{\mathrm{b}}$ & $9.95 \pm 0.37^{\mathrm{b}}$ & $8.45 \pm 0.68^{\mathrm{b}}$ & $8.64 \pm 0.85^{\mathrm{b}}$ \\
& 60 & $7.77 \pm 0.48^{\mathrm{a}}$ & $11.95 \pm 0.57^{\mathrm{a}}$ & $10.11 \pm 0.63^{\mathrm{a}}$ & $9.75 \pm 0.72^{\mathrm{a}}$ & $8.28 \pm 0.14^{\mathrm{a}}$ & $8.35 \pm 1.75^{\mathrm{a}}$ \\
\hline
\end{tabular}

*The values in the same column having similar superscripts do not differ significantly $(\mathrm{p}<0.05)$

Table 4. Manganese content ( $\mathrm{mg} / \mathrm{l00g}$ ) in smoked hilsa during storage period.

\begin{tabular}{|c|c|c|c|c|c|c|c|}
\hline Treatments & Days & Chandpur & Barisal & Patuakhali & Bhola & Shariatpur & Cox's Bazar \\
\hline \multirow{5}{*}{ Control } & 0 (fresh) & $10.70 \pm 2.87^{\mathrm{e}}$ & $10.95 \pm 0.68^{\mathrm{e}}$ & $21.58 \pm 0.48^{\mathrm{e}}$ & $20.97 \pm 1.94^{\mathrm{e}}$ & $10.96 \pm 0.87^{\mathrm{e}}$ & $13.41 \pm 0.69^{\mathrm{e}}$ \\
\hline & 15 & $10.36 \pm 0.98^{\mathrm{d}}$ & $10.06 \pm 0.75^{\mathrm{d}}$ & $20.88 \pm 0.97^{\mathrm{d}}$ & $20.13 \pm 0.78^{d}$ & $10.23 \pm 0.58^{\mathrm{d}}$ & $12.87 \pm 0.48^{\mathrm{d}}$ \\
\hline & 30 & $8.99 \pm 0.96^{\mathrm{c}}$ & $9.35 \pm 0.85^{\mathrm{c}}$ & $19.03 \pm 0.86^{\mathrm{c}}$ & $19.32 \pm 0.94^{\mathrm{c}}$ & $9.67 \pm 0.96^{\mathrm{c}}$ & $11.54 \pm 0.63^{\mathrm{c}}$ \\
\hline & 45 & $8.35 \pm 1.85^{\mathrm{b}}$ & $8.92 \pm 0.86^{\mathrm{b}}$ & $18.37 \pm 0.64^{b}$ & $18.56 \pm 0.75^{b}$ & $8.79 \pm 0.79^{b}$ & $10.78 \pm 0.76^{b}$ \\
\hline & 60 & $7.89 \pm 0.75^{\mathrm{a}}$ & $8.21 \pm 0.25^{\mathrm{a}}$ & $17.80 \pm 0.38^{\mathrm{a}}$ & $17.67 \pm 0.27^{\mathrm{a}}$ & $8.02 \pm 0.48^{\mathrm{a}}$ & $10.32 \pm 0.98^{\mathrm{a}}$ \\
\hline \multirow{5}{*}{ Lemon } & 0 (fresh) & $13.33 \pm 0.94^{\mathrm{e}}$ & $10.71 \pm 0.63^{\mathrm{e}}$ & $17.71 \pm 0.83^{\mathrm{e}}$ & $12.80 \pm 0.42^{\mathrm{e}}$ & $11.88 \pm 0.46^{\mathrm{e}}$ & $11.51 \pm 0.48^{\mathrm{e}}$ \\
\hline & 15 & $12.59 \pm 0.58^{\mathrm{d}}$ & $10.32 \pm 0.47^{\mathrm{d}}$ & $17.13 \pm 0.28^{\mathrm{d}}$ & $11.65 \pm 0.53^{\mathrm{d}}$ & $11.33 \pm 0.47^{\mathrm{d}}$ & $11.03 \pm 0.84^{\mathrm{d}}$ \\
\hline & 30 & $11.65 \pm 0.95^{\mathrm{c}}$ & $9.86 \pm 0.64^{c}$ & $16.79 \pm 1.75^{\mathrm{c}}$ & $10.23 \pm 0.35^{\mathrm{c}}$ & $10.48 \pm 0.74^{\mathrm{c}}$ & $10.49 \pm 0.85^{\mathrm{c}}$ \\
\hline & 45 & $9.32 \pm 0.74^{\mathrm{b}}$ & $9.43 \pm 0.75^{b}$ & $16.32 \pm 0.74^{\mathrm{b}}$ & $9.02 \pm 0.49^{b}$ & $9.78 \pm 0.69^{\mathrm{b}}$ & $9.45 \pm 0.73^{b}$ \\
\hline & 60 & $8.78 \pm 0.26^{\mathrm{a}}$ & $8.54 \pm 0.16^{\mathrm{a}}$ & $15.62 \pm 1.96^{\mathrm{a}}$ & $8.54 \pm 0.38^{\mathrm{a}}$ & $9.04 \pm 0.85^{\mathrm{a}}$ & $8.97 \pm 0.63^{\mathrm{a}}$ \\
\hline \multirow{5}{*}{ Mustard } & 0 (fresh) & $12.37 \pm 0.75^{\mathrm{e}}$ & $11.95 \pm 0.53^{\mathrm{e}}$ & $13.53 \pm 0.97^{\mathrm{e}}$ & $19.56 \pm 0.85^{\mathrm{e}}$ & $12.68 \pm 0.94^{\mathrm{e}}$ & $11.83 \pm 0.68^{\mathrm{e}}$ \\
\hline & 15 & $11.68 \pm 0.83^{\mathrm{d}}$ & $11.11 \pm 0.68^{\mathrm{d}}$ & $12.68 \pm 0.36^{\mathrm{d}}$ & $19.04 \pm 0.76^{\mathrm{d}}$ & $12.09 \pm 0.57^{\mathrm{d}}$ & $10.54 \pm 0.34^{\mathrm{d}}$ \\
\hline & 30 & $10.69 \pm 0.68^{\mathrm{c}}$ & $10.58 \pm 0.63^{\mathrm{c}}$ & $11.76 \pm 0.95^{\mathrm{c}}$ & $18.79 \pm 0.86^{\mathrm{c}}$ & $11.58 \pm 0.48^{\mathrm{c}}$ & $9.74 \pm 0.47^{\mathrm{c}}$ \\
\hline & 45 & $9.87 \pm 0.85^{\mathrm{b}}$ & $10.34 \pm 0.75^{b}$ & $10.98 \pm 0.48^{\mathrm{b}}$ & $18.09 \pm 1.11^{\mathrm{b}}$ & $10.89 \pm 0.43^{b}$ & $9.03 \pm 1.76^{b}$ \\
\hline & 60 & $9.23 \pm 0.94^{\mathrm{a}}$ & $9.99 \pm 0.36^{\mathrm{a}}$ & $10.43 \pm 0.96^{\mathrm{a}}$ & $17.84 \pm 0.64^{\mathrm{a}}$ & $10.43 \pm 0.73^{\mathrm{a}}$ & $8.56 \pm 0.79^{\mathrm{a}}$ \\
\hline \multirow{5}{*}{ Garlic } & 0 (fresh) & $11.76 \pm 0.95^{\mathrm{e}}$ & $11.46 \pm 0.53^{\mathrm{e}}$ & $12.92 \pm 0.97^{\mathrm{e}}$ & $14.40 \pm 0.67^{\mathrm{e}}$ & $12.51 \pm 0.59^{\mathrm{e}}$ & $12.79 \pm 0.64^{\mathrm{e}}$ \\
\hline & 15 & $10.36 \pm 0.97^{\mathrm{d}}$ & $10.97 \pm 0.98^{\mathrm{d}}$ & $12.32 \pm 0.56^{\mathrm{d}}$ & $14.13 \pm 2.67^{\mathrm{d}}$ & $12.05 \pm 0.58^{\mathrm{d}}$ & $11.76 \pm 0.57^{\mathrm{d}}$ \\
\hline & 30 & $9.94 \pm 0.98^{c}$ & $10.52 \pm 0.56^{\mathrm{c}}$ & $11.45 \pm 0.58^{\mathrm{c}}$ & $13.87 \pm 0.84^{\mathrm{c}}$ & $11.93 \pm 1.43^{\mathrm{c}}$ & $10.98 \pm 0.54^{\mathrm{c}}$ \\
\hline & 45 & $9.34 \pm 1.56^{\mathrm{b}}$ & $10.34 \pm 0.38^{b}$ & $10.23 \pm 0.33^{b}$ & $12.45 \pm 0.67^{b}$ & $11.67 \pm 0.76^{\mathrm{b}}$ & $10.57 \pm 0.4^{b}$ \\
\hline & 60 & $8.87 \pm 0.64^{\mathrm{a}}$ & $10.01 \pm 0.97^{\mathrm{a}}$ & $9.87 \pm 0.47^{\mathrm{a}}$ & $11.23 \pm 0.46^{\mathrm{a}}$ & $11.06 \pm 0.15^{\mathrm{a}}$ & $10.22 \pm 0.87^{\mathrm{a}}$ \\
\hline
\end{tabular}

*The values in the same column having similar superscripts do not differ significantly $(\mathrm{p}<0.05)$

Table 5. Copper content (mg/100g) in smoked hilsa during storage period.

\begin{tabular}{|c|c|c|c|c|c|c|c|}
\hline Treatments & Days & Chandpur & Barisal & Patuakhali & Bhola & Shariatpur & Cox's Bazar \\
\hline \multirow{5}{*}{ Control } & 0 (fresh) & $1.68 \pm 0.11^{\mathrm{e}}$ & $1.69 \pm 0.14^{\mathrm{e}}$ & $1.91 \pm 0.05^{\mathrm{e}}$ & $1.85 \pm 0.16^{\mathrm{e}}$ & $1.63 \pm 0.17^{\mathrm{e}}$ & $3.15 \pm 0.16^{\mathrm{e}}$ \\
\hline & 15 & $1.63 \pm 0.12^{\mathrm{d}}$ & $1.51 \pm 0.14^{\mathrm{d}}$ & $1.67 \pm 0.15^{\mathrm{d}}$ & $1.78 \pm 0.15^{\mathrm{d}}$ & $1.57 \pm 0.33^{\mathrm{d}}$ & $3.02 \pm 0.15^{\mathrm{d}}$ \\
\hline & 30 & $1.54 \pm 0.13^{\mathrm{c}}$ & $1.34 \pm 0.21^{\mathrm{c}}$ & $1.48 \pm 0.17^{\mathrm{c}}$ & $1.56 \pm 0.05^{\mathrm{c}}$ & $1.32 \pm 0.26^{\mathrm{c}}$ & $2.87 \pm 0.09^{c}$ \\
\hline & 45 & $1.34 \pm 0.09^{\mathrm{b}}$ & $1.12 \pm 0.09^{\mathrm{b}}$ & $1.32 \pm 0.21^{\mathrm{b}}$ & $1.34 \pm 0.09^{\mathrm{b}}$ & $1.11 \pm 0.06^{\mathrm{b}}$ & $2.56 \pm 0.12^{\mathrm{b}}$ \\
\hline & 60 & $1.21 \pm 0.06^{\mathrm{a}}$ & $0.98 \pm 0.14^{\mathrm{a}}$ & $1.04 \pm 0.24^{\mathrm{a}}$ & $1.16 \pm 0.03^{\mathrm{a}}$ & $0.90 \pm 0.05^{\mathrm{a}}$ & $2.13 \pm 0.21^{\mathrm{a}}$ \\
\hline \multirow{4}{*}{ Lemon } & 0 (fresh) & $1.72 \pm 0.13^{\mathrm{e}}$ & $1.92 \pm 0.13^{\mathrm{e}}$ & $1.58 \pm 0.08^{\mathrm{e}}$ & $2.22 \pm 0.17^{\mathrm{e}}$ & $1.79 \pm 0.19^{\mathrm{e}}$ & $2.15 \pm 0.12^{\mathrm{e}}$ \\
\hline & 30 & $1.53 \pm 0.09^{c}$ & $1.53 \pm 0.05^{\mathrm{c}}$ & $1.43 \pm 0.21^{\mathrm{c}}$ & $2.11 \pm 0.07^{\mathrm{c}}$ & $1.56 \pm 0.05^{\mathrm{c}}$ & $1.67 \pm 0.11^{\mathrm{c}}$ \\
\hline & 45 & $1.46 \pm 0.05^{b}$ & $1.45 \pm 0.17^{\mathrm{b}}$ & $1.21 \pm 0.18^{\mathrm{b}}$ & $1.83 \pm 0.05^{\mathrm{b}}$ & $1.34 \pm 0.38^{\mathrm{b}}$ & $1.56 \pm 0.12^{\mathrm{b}}$ \\
\hline & 60 & $1.32 \pm 0.07^{\mathrm{a}}$ & $1.23 \pm 0.23^{\mathrm{a}}$ & $1.09 \pm 0.08^{\mathrm{a}}$ & $1.71 \pm 0.02^{\mathrm{a}}$ & $1.29 \pm 0.18^{\mathrm{a}}$ & $1.44 \pm 0.31^{\mathrm{a}}$ \\
\hline \multirow{4}{*}{ Mustard } & 0 (fresh) & $1.74 \pm 0.04^{\mathrm{e}}$ & $2.20 \pm 0.05^{\mathrm{e}}$ & $2.03 \pm 0.07^{\mathrm{e}}$ & $1.73 \pm 0.17^{\mathrm{e}}$ & $1.74 \pm 0.11^{\mathrm{e}}$ & $1.88 \pm 0.09^{\mathrm{e}}$ \\
\hline & 15 & $1.64 \pm 0.06^{\mathrm{d}}$ & $1.97 \pm 0.13^{\mathrm{d}}$ & $1.92 \pm 0.15^{\mathrm{d}}$ & $1.58 \pm 0.23^{\mathrm{d}}$ & $1.64 \pm 0.07^{\mathrm{d}}$ & $1.69 \pm 0.15^{\mathrm{d}}$ \\
\hline & 30 & $1.56 \pm 0.08^{c}$ & $1.87 \pm 0.15^{\mathrm{c}}$ & $1.89 \pm 0.09^{c}$ & $1.44 \pm 0.19^{c}$ & $1.51 \pm 0.05^{\mathrm{c}}$ & $1.57 \pm 0.17^{\mathrm{c}}$ \\
\hline & 45 & $1.36 \pm 0.13^{b}$ & $1.76 \pm 0.13^{b}$ & $1.77 \pm 0.14^{\mathrm{b}}$ & $1.37 \pm 0.05^{\mathrm{b}}$ & $1.37 \pm 0.16^{\mathrm{b}}$ & $1.44 \pm 0.04^{\mathrm{b}}$ \\
\hline \multirow{5}{*}{ Garlic } & 0 (fresh) & $1.64 \pm 0.15^{\mathrm{e}}$ & $2.13 \pm 0.06^{\mathrm{e}}$ & $2.66 \pm 0.32^{\mathrm{e}}$ & $1.28 \pm 0.12^{\mathrm{e}}$ & $1.73 \pm 0.03^{\mathrm{e}}$ & $1.93 \pm 0.21^{\mathrm{e}}$ \\
\hline & 15 & $1.57 \pm 0.21^{\mathrm{d}}$ & $2.07 \pm 0.14^{d}$ & $2.55 \pm 0.06^{\mathrm{d}}$ & $1.19 \pm 0.19^{d}$ & $1.66 \pm 0.17^{d}$ & $1.81 \pm 0.13^{\mathrm{d}}$ \\
\hline & 30 & $1.46 \pm 0.14^{\mathrm{c}}$ & $2.02 \pm 0.16^{\mathrm{c}}$ & $2.39 \pm 0.14^{\mathrm{c}}$ & $1.13 \pm 0.20^{\mathrm{c}}$ & $1.54 \pm 0.06^{\mathrm{c}}$ & $1.67 \pm 0.18^{\mathrm{c}}$ \\
\hline & 45 & $1.26 \pm 0.06^{\mathrm{b}}$ & $1.99 \pm 0.21^{\mathrm{b}}$ & $2.29 \pm 0.19^{b}$ & $1.02 \pm 0.04^{\mathrm{b}}$ & $1.44 \pm 0.30^{\mathrm{b}}$ & $1.56 \pm 0.17^{\mathrm{b}}$ \\
\hline & 60 & $1.18 \pm 0.18^{\mathrm{a}}$ & $1.87 \pm 0.08^{\mathrm{a}}$ & $2.02 \pm 0.05^{\mathrm{a}}$ & $0.92 \pm 0.18^{\mathrm{a}}$ & $1.35 \pm 0.07^{\mathrm{a}}$ & $1.41 \pm 0.04^{\mathrm{a}}$ \\
\hline
\end{tabular}

*The values in the same column having similar superscripts do not differ significantly $(\mathrm{p}<0.05)$ 
Table 6. Magnesium content ( $\mathrm{mg} / 100 \mathrm{~g})$ in smoked hilsa during storage period.

\begin{tabular}{|c|c|c|c|c|c|c|c|}
\hline Treatments & Days & Chandpur & Barisal & Patuakhali & Bhola & Shariatpur & Cox's Bazar \\
\hline \multirow{5}{*}{ Control } & 0 (fresh) & $57.59 \pm 4.87^{\mathrm{e}}$ & $52.81 \pm 2.76^{\mathrm{e}}$ & $58.22 \pm 6.54^{\mathrm{e}}$ & $45.10 \pm 0.98^{\mathrm{e}}$ & $60.54 \pm 10.66^{\mathrm{e}}$ & $48.19 \pm 1.09^{\mathrm{e}}$ \\
\hline & 15 & $53.98 \pm 9.78^{d}$ & $50.25 \pm 4.68^{d}$ & $54.37 \pm 8.76^{\mathrm{d}}$ & $43.25 \pm 0.78^{d}$ & $55.48 \pm 13.46^{\mathrm{d}}$ & $47.42 \pm 6.57^{\mathrm{d}}$ \\
\hline & 30 & $51.34 \pm 8.45^{\mathrm{c}}$ & $47.17 \pm 5.98^{c}$ & $48.38 \pm 5.64^{c}$ & $41.42 \pm 0.65^{c}$ & $49.72 \pm 0.98^{c}$ & $46.42 \pm 4.43^{c}$ \\
\hline & 45 & $48.47 \pm 6.63^{b}$ & $45.23 \pm 6.98^{b}$ & $46.35 \pm 3.67^{b}$ & $39.45 \pm 4.87^{b}$ & $45.78 \pm 1.76^{\mathrm{b}}$ & $43.48 \pm 8.34^{b}$ \\
\hline & 60 & $46.34 \pm 9.29^{\mathrm{a}}$ & $43.37 \pm 7.47^{\mathrm{a}}$ & $42.43 \pm 5.32^{\mathrm{a}}$ & $36.31 \pm 1.85^{\mathrm{a}}$ & $41.98 \pm 4.87^{\mathrm{a}}$ & $40.98 \pm 9.87^{\mathrm{a}}$ \\
\hline \multirow{5}{*}{ Lemon } & 0 (fresh) & $61.22 \pm 0.74^{\mathrm{e}}$ & $55.88 \pm 3.58^{\mathrm{e}}$ & $61.95 \pm 7.65^{\mathrm{e}}$ & $48.23 \pm 9.58^{\mathrm{e}}$ & $62.92 \pm 5.50^{\mathrm{e}}$ & $51.63 \pm 3.52^{\mathrm{e}}$ \\
\hline & 15 & $60.47 \pm 9.53^{\mathrm{d}}$ & $54.13 \pm 9.54^{\mathrm{d}}$ & $59.87 \pm 4.98^{\mathrm{d}}$ & $47.14 \pm 5.32^{\mathrm{d}}$ & $61.34 \pm 4.35^{\mathrm{d}}$ & $50.55 \pm 7.43^{d}$ \\
\hline & 30 & $59.31 \pm 4.87^{\mathrm{c}}$ & $53.75 \pm 7.58^{c}$ & $58.32 \pm 8.75^{\mathrm{c}}$ & $46.72 \pm 2.76^{\mathrm{c}}$ & $60.15 \pm 7.65^{c}$ & $49.32 \pm 8.09^{c}$ \\
\hline & 45 & $58.39 \pm 10.05^{\mathrm{b}}$ & $52.79 \pm 3.70^{\mathrm{b}}$ & $56.48 \pm 2.08^{b}$ & $43.21 \pm 2.79^{b}$ & $58.32 \pm 4.35^{\mathrm{b}}$ & $47.52 \pm 2.98^{b}$ \\
\hline & 60 & $57.23 \pm 0.63^{\mathrm{a}}$ & $51.24 \pm 5.79^{\mathrm{a}}$ & $54.71 \pm 2.16^{\mathrm{a}}$ & $41.95 \pm 6.87^{\mathrm{a}}$ & $56.83 \pm 4.43^{\mathrm{a}}$ & $46.23 \pm 7.57^{\mathrm{a}}$ \\
\hline \multirow{5}{*}{ Mustard } & 0 (fresh) & $63.90 \pm 8.89^{\mathrm{e}}$ & $63.90 \pm 1.84^{\mathrm{e}}$ & $63.08 \pm 5.43^{\mathrm{e}}$ & $50.85 \pm 4.09^{\mathrm{e}}$ & $63.59 \pm 6.54^{\mathrm{e}}$ & $54.77 \pm 8.09^{\mathrm{e}}$ \\
\hline & 15 & $61.46 \pm 3.79^{d}$ & $62.15 \pm 2.74^{\mathrm{d}}$ & $62.34 \pm 8.76^{\mathrm{d}}$ & $49.32 \pm 8.97^{\mathrm{d}}$ & $61.89 \pm 6.43^{\mathrm{d}}$ & $52.67 \pm 9.76^{d}$ \\
\hline & 30 & $60.32 \pm 2.96^{\mathrm{c}}$ & $58.92 \pm 6.57^{\mathrm{c}}$ & $60.87 \pm 6.75^{\mathrm{c}}$ & $47.31 \pm 6.54^{\mathrm{c}}$ & $59.07 \pm 1.25^{\mathrm{c}}$ & $51.56 \pm 11.87^{\circ}$ \\
\hline & 45 & $59.62 \pm 4.89^{b}$ & $55.47 \pm 9.84^{b}$ & $59.43 \pm 7.64^{b}$ & $45.63 \pm 7.65^{b}$ & $57.85 \pm 2.63^{b}$ & $50.76 \pm 5.76^{b}$ \\
\hline & 60 & $57.89 \pm 6.47^{\mathrm{a}}$ & $53.56 \pm 8.47^{\mathrm{a}}$ & $56.86 \pm 6.53^{\mathrm{a}}$ & $44.65 \pm 4.53^{\mathrm{a}}$ & $56.47 \pm 4.87^{\mathrm{a}}$ & $48.72 \pm 3.98^{\mathrm{a}}$ \\
\hline \multirow{5}{*}{ Garlic } & 0 (fresh) & $64.10 \pm 9.05^{\mathrm{e}}$ & $64.10 \pm 6.53^{\mathrm{e}}$ & $64.82 \pm 5.45^{\mathrm{e}}$ & $51.30 \pm 2.76^{\mathrm{e}}$ & $63.95 \pm 1.67^{\mathrm{e}}$ & $55.10 \pm 2.76^{\mathrm{e}}$ \\
\hline & 15 & $63.77 \pm 7.95^{\mathrm{d}}$ & $63.43 \pm 2.76^{\mathrm{d}}$ & $63.21 \pm 3.09^{\mathrm{d}}$ & $49.99 \pm 4.98^{\mathrm{d}}$ & $63.24 \pm 3.65^{\mathrm{d}}$ & $53.99 \pm 2.76^{\mathrm{d}}$ \\
\hline & 30 & $62.76 \pm 3.24^{\mathrm{c}}$ & $61.11 \pm 3.87^{\mathrm{c}}$ & $61.56 \pm 9.06^{\mathrm{c}}$ & $48.95 \pm 7.53^{\mathrm{c}}$ & $62.86 \pm 6.98^{c}$ & $52.78 \pm 2.09^{c}$ \\
\hline & 45 & $60.12 \pm 1.86^{\mathrm{b}}$ & $60.13 \pm 5.66^{\mathrm{b}}$ & $60.78 \pm 10.87^{b}$ & $47.67 \pm 2.65^{b}$ & $62.31 \pm 2.47^{\mathrm{b}}$ & $51.91 \pm 7.65^{\mathrm{b}}$ \\
\hline & 60 & $58.51 \pm 2.65^{\mathrm{a}}$ & $58.95 \pm 1.68^{\mathrm{a}}$ & $59.74 \pm 12.76^{\mathrm{a}}$ & $46.87 \pm 1.97^{\mathrm{a}}$ & $61.61 \pm 5.43^{\mathrm{a}}$ & $51.65 \pm 1.98^{\mathrm{a}}$ \\
\hline
\end{tabular}

*The values in the same column having similar superscripts do not differ significantly $(\mathrm{p}<0.05)$

Table 7. Zinc content ( $\mathrm{mg} / \mathrm{l00g})$ in smoked hilsa during storage period.

\begin{tabular}{|c|c|c|c|c|c|c|c|}
\hline Treatments & Days & Chandpur & Barisal & Patuakhali & Bhola & Shariatpur & Cox's Bazar \\
\hline \multirow{5}{*}{ Control } & 0 (fresh) & $2.69 \pm 0.12^{\mathrm{e}}$ & $2.70 \pm 0.16^{\mathrm{e}}$ & $2.92 \pm 0.09^{\mathrm{e}}$ & $2.86 \pm 0.13^{\mathrm{e}}$ & $2.64 \pm 0.13^{\mathrm{e}}$ & $2.97 \pm 0.10^{\mathrm{e}}$ \\
\hline & 15 & $1.64 \pm 0.13^{d}$ & $1.52 \pm 0.11^{\mathrm{d}}$ & $1.68 \pm 0.11^{\mathrm{d}}$ & $1.79 \pm 0.11^{\mathrm{d}}$ & $1.58 \pm 0.31^{\mathrm{d}}$ & $2.93 \pm 0.11^{\mathrm{d}}$ \\
\hline & 30 & $1.55 \pm 0.14^{\mathrm{c}}$ & $1.37 \pm 0.23^{\mathrm{c}}$ & $1.49 \pm 0.14^{\mathrm{c}}$ & $1.55 \pm 0.02^{\mathrm{c}}$ & $1.38 \pm 0.28^{c}$ & $2.89 \pm 0.04^{\mathrm{c}}$ \\
\hline & 45 & $1.34 \pm 0.07^{\mathrm{b}}$ & $1.12 \pm 0.01^{\mathrm{b}}$ & $1.32 \pm 0.22^{\mathrm{b}}$ & $1.34 \pm 0.03^{\mathrm{b}}$ & $1.11 \pm 0.05^{\mathrm{b}}$ & $2.56 \pm 0.11^{\mathrm{b}}$ \\
\hline & 60 & $1.23 \pm 0.05^{\mathrm{a}}$ & $0.99 \pm 0.13^{\mathrm{a}}$ & $1.05 \pm 0.21^{\mathrm{a}}$ & $1.17 \pm 0.09^{\mathrm{a}}$ & $0.92 \pm 0.02^{\mathrm{a}}$ & $2.17 \pm 0.23^{\mathrm{a}}$ \\
\hline \multirow{5}{*}{ Lemon } & 0 (fresh) & $3.72 \pm 0.11^{\mathrm{e}}$ & $3.92 \pm 0.14^{\mathrm{e}}$ & $3.58 \pm 0.09^{\mathrm{e}}$ & $3.22 \pm 0.12^{\mathrm{e}}$ & $3.79 \pm 0.11^{\mathrm{e}}$ & $3.15 \pm 0.09^{\mathrm{e}}$ \\
\hline & 15 & $1.67 \pm 0.14^{\mathrm{d}}$ & $1.79 \pm 0.06^{\mathrm{d}}$ & $1.48 \pm 0.13^{\mathrm{d}}$ & $2.18 \pm 0.11^{\mathrm{d}}$ & $1.68 \pm 0.26^{\mathrm{d}}$ & $1.93 \pm 0.03^{\mathrm{d}}$ \\
\hline & 30 & $1.53 \pm 0.07^{\mathrm{c}}$ & $1.53 \pm 0.03^{\mathrm{c}}$ & $1.43 \pm 0.24^{\mathrm{c}}$ & $2.11 \pm 0.08^{c}$ & $1.56 \pm 0.02^{\mathrm{c}}$ & $1.67 \pm 0.10^{\mathrm{c}}$ \\
\hline & 45 & $1.43 \pm 0.09^{b}$ & $1.47 \pm 0.13^{\mathrm{b}}$ & $1.25 \pm 0.19^{\mathrm{b}}$ & $1.87 \pm 0.06^{\mathrm{b}}$ & $1.38 \pm 0.34^{\mathrm{b}}$ & $1.57 \pm 0.17^{\mathrm{b}}$ \\
\hline & 60 & $1.33 \pm 0.03^{\mathrm{a}}$ & $1.24 \pm 0.24^{\mathrm{a}}$ & $1.11 \pm 0.09^{\mathrm{a}}$ & $1.73 \pm 0.03^{\mathrm{a}}$ & $1.26 \pm 0.13^{\mathrm{a}}$ & $1.44 \pm 0.21^{\mathrm{a}}$ \\
\hline \multirow{5}{*}{ Mustard } & 0 (fresh) & $2.76 \pm 0.06^{\mathrm{e}}$ & $2.24 \pm 0.08^{\mathrm{e}}$ & $2.06 \pm 0.05^{\mathrm{e}}$ & $2.77 \pm 0.19^{\mathrm{e}}$ & $2.75 \pm 0.12^{\mathrm{e}}$ & $2.82 \pm 0.19^{\mathrm{e}}$ \\
\hline & 15 & $1.62 \pm 0.07^{\mathrm{d}}$ & $1.95 \pm 0.13^{\mathrm{d}}$ & $1.93 \pm 0.10^{\mathrm{d}}$ & $1.83 \pm 0.21^{\mathrm{d}}$ & $1.66 \pm 0.09^{d}$ & $1.64 \pm 0.25^{d}$ \\
\hline & 30 & $1.57 \pm 0.09^{c}$ & $1.88 \pm 0.16^{\mathrm{c}}$ & $1.90 \pm 0.04^{\mathrm{c}}$ & $1.46 \pm 0.12^{\mathrm{c}}$ & $1.53 \pm 0.03^{c}$ & $1.56 \pm 0.27^{\mathrm{c}}$ \\
\hline & 45 & $1.36 \pm 0.12^{\mathrm{b}}$ & $1.76 \pm 0.12^{b}$ & $1.77 \pm 0.19^{b}$ & $1.37 \pm 0.09^{\mathrm{b}}$ & $1.37 \pm 0.17^{b}$ & $1.44 \pm 0.14^{\mathrm{b}}$ \\
\hline & 60 & $1.28 \pm 0.11^{\mathrm{a}}$ & $1.57 \pm 0.08^{\mathrm{a}}$ & $1.68 \pm 0.03^{\mathrm{a}}$ & $1.29 \pm 0.04^{\mathrm{a}}$ & $1.23 \pm 0.23^{\mathrm{a}}$ & $1.26 \pm 0.13^{\mathrm{a}}$ \\
\hline \multirow{5}{*}{ Garlic } & 0 (fresh) & $2.65 \pm 0.11^{\mathrm{e}}$ & $2.16 \pm 0.09^{\mathrm{e}}$ & $2.69 \pm 0.30^{\mathrm{e}}$ & $2.29 \pm 0.11^{\mathrm{e}}$ & $2.77 \pm 0.04^{\mathrm{e}}$ & $2.90 \pm 0.01^{\mathrm{e}}$ \\
\hline & 15 & $1.57 \pm 0.22^{\mathrm{d}}$ & $2.07 \pm 0.17^{\mathrm{d}}$ & $2.55 \pm 0.03^{\mathrm{d}}$ & $1.19 \pm 0.13^{\mathrm{d}}$ & $1.66 \pm 0.18^{\mathrm{d}}$ & $1.81 \pm 0.23^{\mathrm{d}}$ \\
\hline & 30 & $1.47 \pm 0.17^{\mathrm{c}}$ & $2.06 \pm 0.19^{c}$ & $2.38 \pm 0.12^{\mathrm{c}}$ & $1.15 \pm 0.22^{\mathrm{c}}$ & $1.58 \pm 0.09^{c}$ & $1.69 \pm 0.08^{\mathrm{c}}$ \\
\hline & 45 & $1.26 \pm 0.05^{\mathrm{b}}$ & $1.99 \pm 0.20^{\mathrm{b}}$ & $2.29 \pm 0.14^{\mathrm{b}}$ & $1.02 \pm 0.07^{\mathrm{b}}$ & $1.44 \pm 0.31^{\mathrm{b}}$ & $1.56 \pm 0.07^{\mathrm{b}}$ \\
\hline & 60 & $1.19 \pm 0.12^{\mathrm{a}}$ & $1.88 \pm 0.07^{\mathrm{a}}$ & $2.01 \pm 0.04^{\mathrm{a}}$ & $0.93 \pm 0.19^{\mathrm{a}}$ & $1.37 \pm 0.06^{\mathrm{a}}$ & $1.46 \pm 0.14^{\mathrm{a}}$ \\
\hline
\end{tabular}

*The values in the same column having similar superscripts do not differ significantly $(\mathrm{p}<0.05)$

\section{Conclusion}

The results of the investigation shows that smoked hilsa is good sources of minerals apart from proteins which are the main nutrient associated with fish. It was observed that processing and storage significantly affected the minearls of smoked fish and their effect varied from regions to regions. It is right to suggest that, the mineral elemental compositions of smoked hilsa is a function of the availability of these elements in their local environment, diet absorptive capability as well as their preferential accumulation. Moreover, the study has also highlighted that the mineral composition of different regions of smoked hilsa may be affected differently by the methods of treatments, preservation and duration of storage.

\section{References}

[1] Glover CN, Hogstrand C (2002) Amino acids of in vivo intestinal zinc absorption in freshwater rainbow trout. Journal of Experimental Biology 205: 151-158.

[2] Sivaperumal P, Sankar TV, Viswanathan Nair PG (2007) Heavy metal concentrations in fish, shellfish and fish products from internal markets of India vis-à-vis international standards. Food Chemistry 102(3): 612-620.

[3] Sikorski ZE (1990) Resources Nutritional Composition and Preservation. Boca Raton, Florida. CRC Press-Inc. pp. 30-52. 
[4] Rodrigo J, Ros G, Priago J, Lopez C, Ortuno J (1998) Proximate and mineral composition of dried salted roes of hake (Merlucciusmerluccius L.) and ling (Molvamolva L.). Food Chemistry 63(2): 221-225.

[5] Alasalvar C, Taylor KDA, Zubcov E, Shahidi F, Alexis M (2002) Differentiation of cultured and wild sea bass (Dicentrar chuslabrax): total lipid content, fatty acid and trace mineral composition. Food Chemistry 79(2): 145-150.

[6] Turhan S, Ustun SN, Altunkaynak B (2004) Effect of cooking methods on total and heme iron contents of anchovy (Engraulisen crasicholus). Food Chemistry 88(2): 169-172.

[7] Adewoye SO, Omotosho JS (1997) Nutrient Composition of some freshwater fishes in Nigeria. Bioscience Research Communications 11 (4): 333-336.

[8] Boyd CE, Davis JA (1978) Concentration of selected element and ash in Bluegill (Lepomis macrochirus) and certain other freshwater fish. Transactions of the American Fisheries Society 6: 862-867.

[9] AOAC (2002) Official Method of Analysis of Association of Official Analytical Chemists 4(7), Bancroft, G.D., USA.

[10] Cole RC, Clucas IJ, Bostock TW, Rogers JF, Stephens AD, Debling GB, Clifford M, Homer W (1982) Fish handling, preservation and processing in the tropics: Part 2. Tropical Development and Research Institute. London.
[11] Olivares M, Pizarro F, Ruz M (2007) New Insights about Iron Bioavailability Inhibition by Zinc. Nutrition 23: 292-295.

[12] Martínez-Alvarez O, Gomez-Guillén MC (2006) Effect of brine salting at different $\mathrm{pH}$ on the functional properties of cod muscle proteins after subsequent dry salting. Food Chemistry 94: 123-129.

[13] Window H, Stein D, Scheldon R, Smith JR (1987) Comparison of trace metal concentrations in muscle of a benthopelagic fish (Cory phaenoides armatus) from the Atlantic and Pacific oceans. Deep sea Research 34 (2): 213220 .

[14] Sikorski ZF, Sunpan B (1992) Preservation of seafood quality. In: Shahidi, F., Botter, J.R. (Eds.). Seafood's Chemistry Processing.

[15] Arannilewa ST, Salawu SO, Sorungbe AA, Olasa lawu BB (2005) Effect of frozen period on the chemical, microbiological and sensory quality of frozen tilapia fish (Sarotherod on galilaleus). African Journal of Biotechnology 4(8): 852-855.

[16] Obuz E, Dikeman ME (2003) Effect of cooking beef muscle from frozen or thawed states on cooking traits palatability. Meat Science 65: 993-997. 